\title{
Energy models towards the retrofitting of the historic built heritage
}

\author{
F. Fatiguso, M. De Fino, E. Cantatore, A. Scioti \\ \& G. De Tommasi \\ Department of Civil, Environmental, Territory, \\ Building Engineering and of Chemistry, Polytechnic of Bari, Italy
}

\begin{abstract}
Scientific community and government institutions have acknowledged energyretrofitting solutions for historic buildings as strategic within the EU "20-20-20" target, provided that they should be developed from the assessment of the local environment. Geographic-climatic conditions of site, historic-morphological development of settlement, typological-architectural configuration of buildings and construction-technical characteristics of components feature that environment, which shapes the domain, where expected performances of requalification products and processes might be defined. The final goal is the challenging and desirable balance among conservation of original material and formal identity, enhancement of present and potential relationships between indoor space and outdoor environment, technological improvement of construction systems. The paper is going to discuss an assessment methodology, applied to the historic sea towns in the Province of Bari, South Italy. Specifically, the methodology focuses on the investigation of all the above-mentioned aspects, also by means of analytical simulations, onsite measurements and thematic maps, in order to develop reliable energy models for historic buildings. The models should support the analysis of the relationships between performances of building sub-systems and efficiency of building system, always taking into account the transformation boundaries of the envelope, towards efficient, compatible and convenient energy-retrofitting solutions.
\end{abstract}

Keywords: historic built heritage, energy assessment models, innovative retrofitting solutions. 


\section{Introduction}

The historic built heritage results from a multi-faceted process, firmly related to the cultural, social and environmental background. In fact, the original concept of the traditional architecture relies on the geographic and climatic conditions of the site, the local construction materials and techniques and the use by the resident community. Furthermore, it develops throughout the centuries, based on regional political targets, management strategies and technological and normative evolution.

Nevertheless, according to a well-established methodological approach [1-6], the energy retrofitting of the historic built heritage should overcome the variety of typological, architectural and technical solutions, even featuring the district scale, in order to address a geo-cluster based vision. Geo-clusters are conceived as "virtual trans-national areas/markets where strong similarities are found, in terms of climate, culture and behavior, construction typologies, economy, energy/resources price policies, technological solutions and building materials applied because of local demand-supply aspects" [7]. Specifically, such a vision aims at developing assessment methods and retrofitting solutions at the macroscale, in order to enhance the "refurbishment rate and depth" of the historic buildings $-30 \%$ of the existing buildings in Europe - by a systemic management of critical key aspects, including analysis and control of actual performances, indoor comfort, involvement of users, scattered supply chain [8].

Within that framework, the European Community has addressed the Horizon 2020 research program, with specific calls - e.g. EE-03-2014: Energy strategies and solutions for deep renovation of historic buildings and EeB-05-2015: Innovative design tools for refurbishing of buildings at district level. In detail, three main targeted areas might be identified: (i) creation of databases on energy and environmental performances of historic buildings, (ii) identification of innovative refurbishment design tools from the district to the building scale, and (iii) definition of libraries of reference solution.

Moreover, as acknowledged by scientific publications [9-12] and research projects $[13,14]$, those targeted areas might rely on the development of energy benchmarking building-types, where all the investigated buildings could be grouped in energy categories and analyzed by energy models. Consequently, an in-depth preliminary qualification - from typological-architectural features of the district to the constructional-technical characteristics of the buildings - is required, in order to shape the domain, where expected performances of requalification products and processes might be defined. The final goal is the challenging and desirable balance among conservation of original material and formal identity, enhancement of present and potential relationships between indoor space and outdoor environment, technological improvement of construction systems.

\section{Methodology}

In the light of the abovementioned aspects, the paper is going to outline a methodology for the energy retrofitting of the historic built heritage, based on the 
identification of the reference geo-cluster, the development of the energy benchmarking building-types and the validation of the compatible refurbishment solutions for scalability and replicability at the macro-scale. Particularly, the paper is going to focus on some results about a sea historic town in Mediterranean climate, as representative pilot-case of the construction materials and techniques of the traditional architecture in Apulia Region, South Italy.

In detail, the methodology comprises three main phases:

Phase I. Identification of the geo-cluster. It is based on collection of geographic and climatic data, simulation of solar radiation and wind exposure, mapping of typologies, materials, construction techniques and historicarchitectural values of all the buildings. All the data, also from onsite experimental measurements and analytical simulations, are acquired according to specific protocols, including informative databases, checklists and platforms - e.g. ArcGIS - in order to enable the crosselaboration of information from different sources and cases.

Phase II. Definition of energy benchmarking. It is based on the taxonomic elaboration of environmental, historic, morphological-typological and constructional-technical characteristics - as gathered in Phase I - in order to create a limited number of models. The models should describe performances and transformation degrees of the envelope components in the historic district. In detail, within the models, all the building subsystems are described in terms of physical performances (e.g. thermal transmittance), so that the influence of each performance on the heating and cooling saving can be evaluated by means of iterative heat transient simulations. On the other hand, the transformation degrees are used in order to develop compatible integration/modification layouts for the envelope components.

Phase III. Selection and validation of compatible retrofitting solutions. It results from the comparison of the integration/modification layouts - as developed in Phase II - with the available traditional and innovative refurbishment products and systems, eventually assessed by cost/benefit and life cycle analyses.

\section{Case study}

The case study, herein presented, is the old town of Molfetta, located $40 \mathrm{~km}$ north from Bari, the main city of Apulia Region. Specifically, the abovementioned methodology phases were carried out, as follows.

\subsection{Identification of the geo-cluster}

Within a GIS platform, several thematic maps were developed, based on data about the historic evolution and the urban arrangement of the district, the morphology and typology of the buildings, the materials and techniques of the envelope components. 
As far as the historic evolution is concerned (Figure 1), the old town is located on Sant'Andrea peninsula, with compact arrangement and well-preserved original character. The early settlement dates back to the Middle Ages, although several additions occurred throughout the years, until the late XIX century. The result is a dense district by the port, with high and solid buildings along the seafront on the north side, as barriers against outside attacks. The peninsula shows elliptical shape: the shortest axe is NNW-SSE oriented, while fourteen small secondary routes branch off it in the opposite direction. The very narrow paths strongly affect the district permeability to solar radiation and wind exposure.

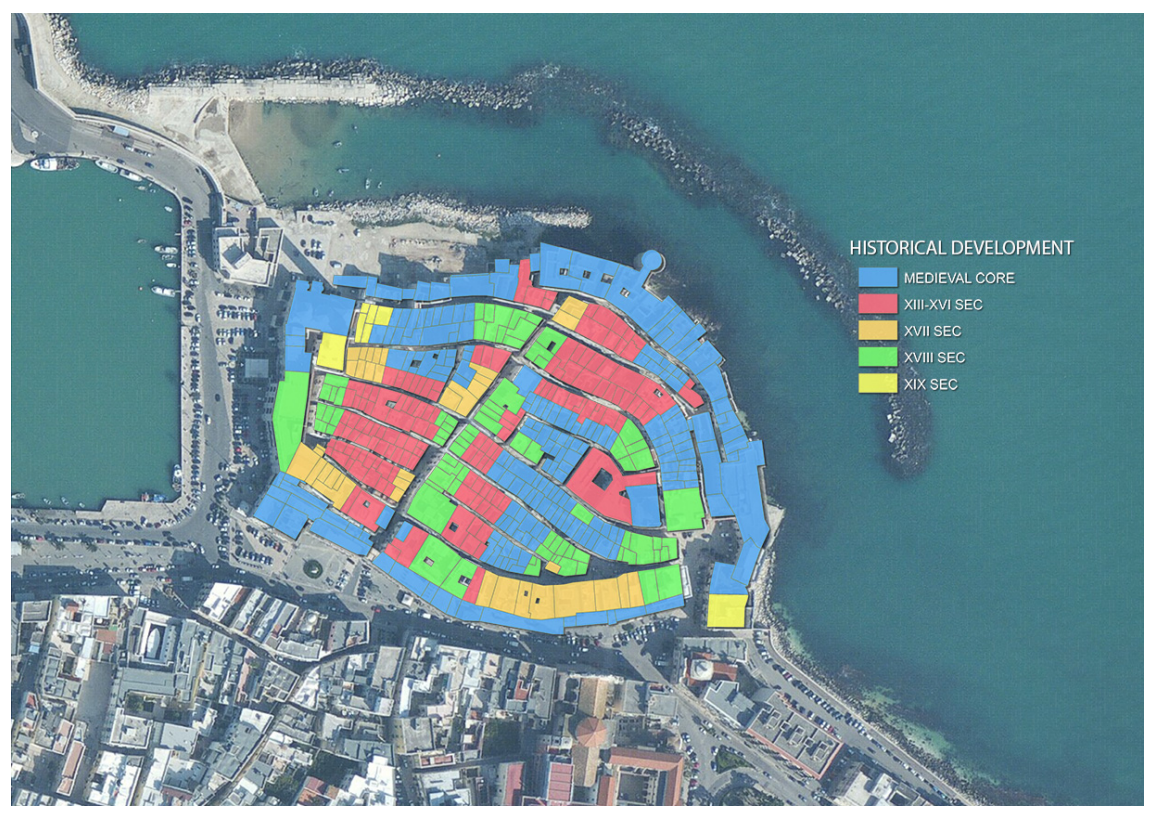

Figure 1: GIS map of historic evolution.

As far as the district morphology and building typology are concerned (Figure 2 and Figure 3), the old town is composed of nineteen blocks, 35-50 meters long. Each block is the aggregation of several aligned buildings, generally 3-5 floors high, with commercial stores at the ground level and private dwellings at the upper levels. Specifically, the dwellings mainly belong to two main categories:

1. "tower house", where the dwelling occupies all the floors of one building and, thus, shows a column of openings on the external façade, with high and narrow street front;

2. "palace house", where the dwelling occupies one floor of two/three buildings and, thus, shows a row of openings on the external façade, with short and large street front. 


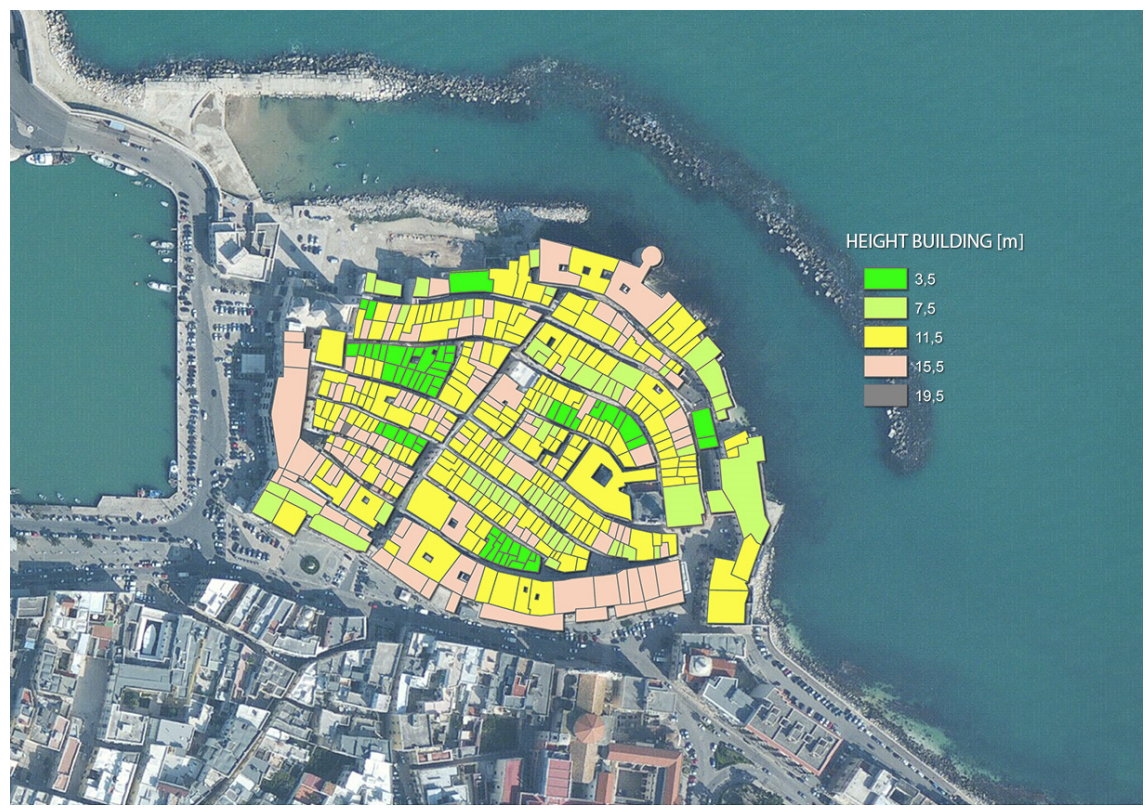

Figure 2: GIS map of building height.

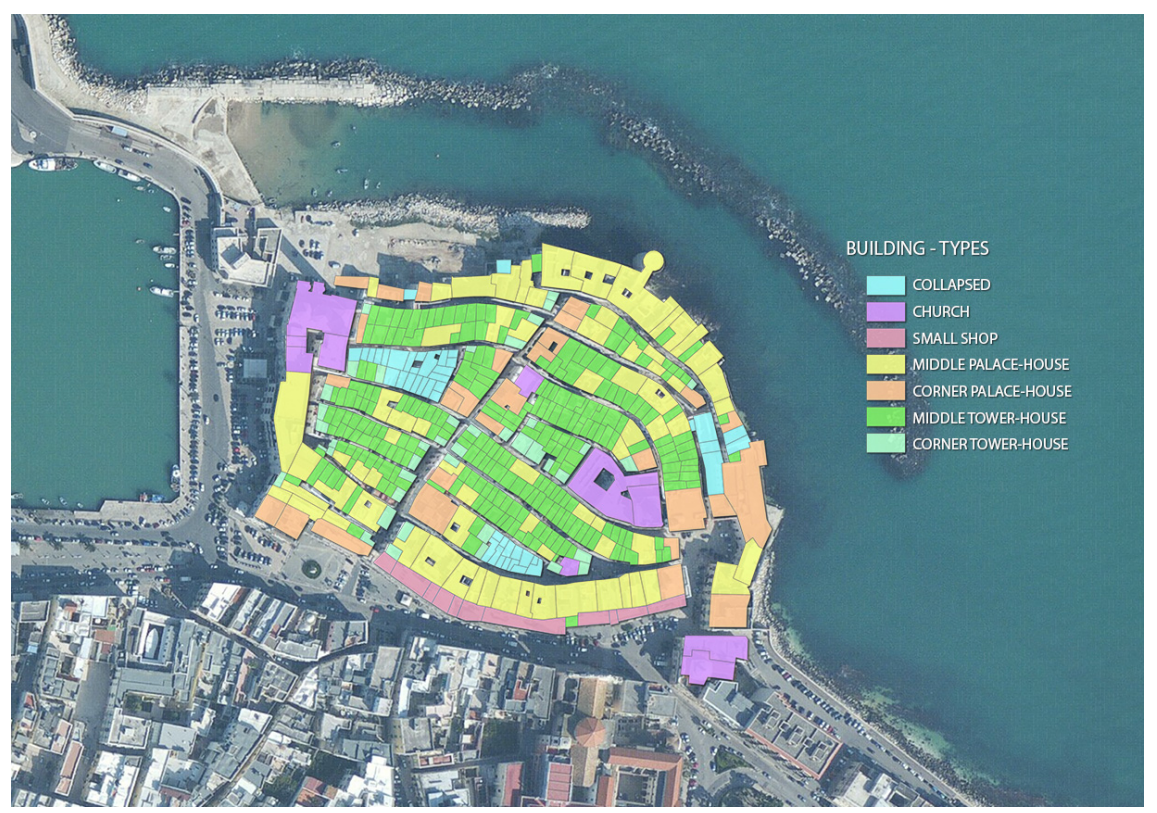

Figure 3: GIS map of building typologies. 
As far as the construction materials and techniques are concerned, the old town is still featured by traditional solutions, as follows:

1. Walls are made out of two outer leaves of local stone squared blocks and an inner cavity filled by mortar mixtures and natural aggregates. They are from $50 \mathrm{~cm}$ to $90 \mathrm{~cm}$ thick. At the ground level, they are generally unplastered, while at the upper levels they are covered by a $3 \mathrm{~cm}$ thick plaster layer;

2. Basement slabs are placed above a layer of stone blocks and gravel as barrier against the dampness. Tiles are generally $5 \mathrm{~cm}$ thick and made out of local stone;

3. Roofs show wooden structure and outer finishing by stone tiles or waterproofing layer;

4. Windows show modern wooden frames and double-glazing.

All the above-mentioned components were classified according to their transformation degree, namely their attitude to be modified without altering their historic and architectural features, as acknowledged by the current scientific debate on the topic [15].

For instance, three degrees were identified and mapped for the roofs (Figure 4):

1. HIGH: for roofs that are severely damaged or collapsed, so that the intervention should concern their reconstruction;

2. MEDIUM: for roofs covered by waterproofing layer, where the design and construction of a compatible finishing might be required;

3. LOW-MEDIUM: for roofs covered by original either/or valuable roof tiles, where the intervention should preserve the formal and material identity of the external finishing, eventually by removal, treatment and reuse.

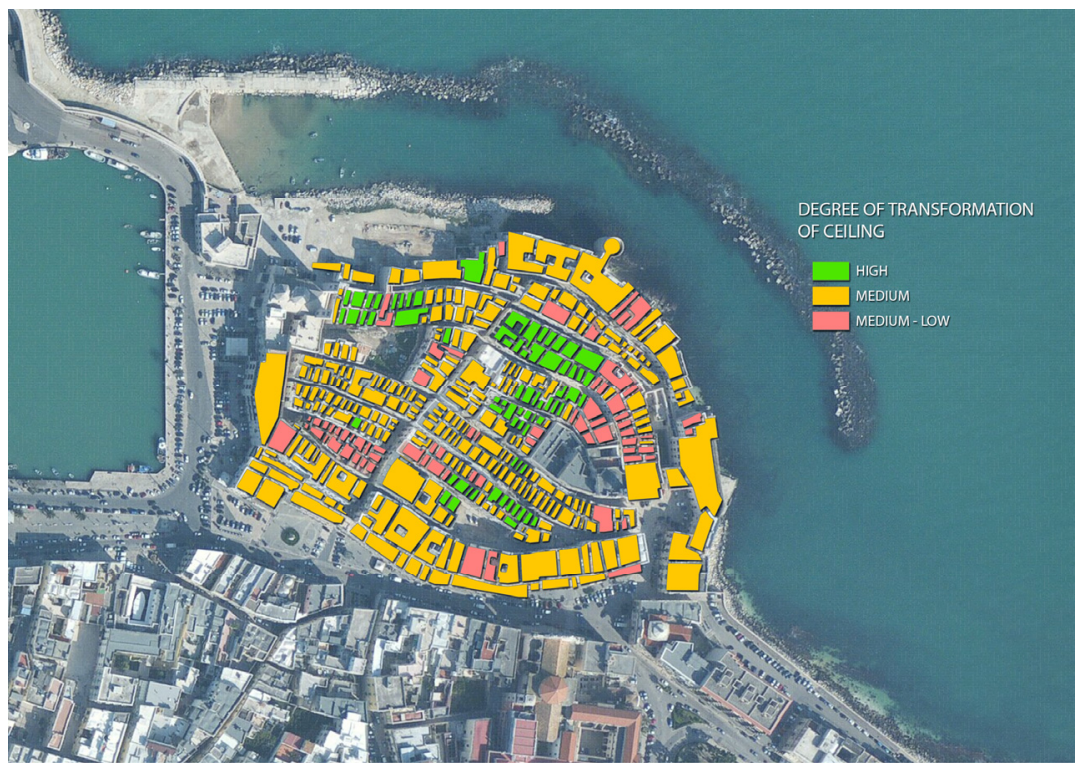

Figure 4: GIS map of transformation degree of roofs. 
Similarly, with reference to the walls (Figure 5):

1. HIGH: for walls that are severely damaged or collapsed, so that the intervention should concern their reconstruction;

2. MEDIUM-HIGH: for plastered walls, so that the design should concern the replacement of the original finishing with compatible layers of high performing materials;

3. LOW: for unplastered walls, where the intervention is quite limited by the conservation requirements.

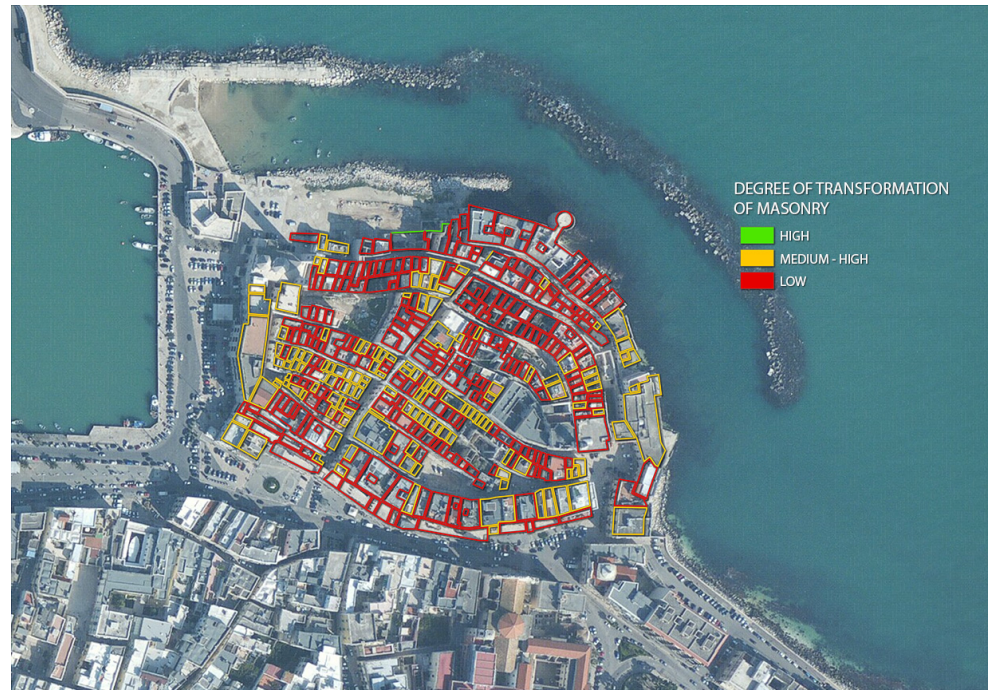

Figure 5: GIS map of transformation degree of walls.

\subsection{Definition of energy benchmarking models}

Based on the data on district morphology and building typology, eight buildingtypes were identified:

A. TOWER HOUSE:

A1. MIDDLE UNIT: the building is within the block and, thus, shows only one front towards the outside;

A2. END UNIT: the building is at the corner of the block and, thus, shows two fronts toward the outside.

B. PALACE HOUSE:

B1. MIDDLE UNIT:

B1.1 FIRST FLOOR: the dwelling is delimited by a commercial unit below and a housing unit above;

B1.2 ROOF FLOOR: the dwelling is delimited by two housing units, both below and above;

B2. END UNIT:

B2.1. FIRST FLOOR;

B2.2. ROOF FLOOR. 
Each building-type might be analysed for two orientation (NE and SW). Thus, starting from that classification, sixteen energy models were developed, based on common dimensions and thermal characteristics of the envelope components.

Two building-types of tower houses are discussed in detail below. Specifically, from the survey of all the buildings belonging to that category, models were built, as follows:

- $\quad 8 \mathrm{~m} \mathrm{x} 4 \mathrm{~m}$ plan with only one street front with windows corresponding to the shortest dimension;

- $\quad 4$ floors with one commercial store at the ground floor and one dwelling with independent entrance and staircase from the street at the upper floors;

- $\quad 13.5 \mathrm{~m}$ height, with $3.5 \mathrm{~m}$ high ground floor and $3 \mathrm{~m}$ high upper floors;

- $\quad$ basement slab made out of a not insulated layer of stone blocks and gravel (thermal transmittance $=2.83 \mathrm{~W} / \mathrm{m}^{2} \mathrm{~K}$ )

- $\quad$ roof made out of wooden beams and slabs, covered by sloping lightweight concrete slab, mortars and stone tiles (overall thickness $=17 \mathrm{~cm}$; thermal transmittance $=1.83 \mathrm{~W} / \mathrm{m}^{2} \mathrm{~K}$ );

- $\quad$ masonry cavity walls, covered by plaster, except from the external surface at the ground floor (overall thickness $=80 \mathrm{~cm}$; thermal transmittance $=2 \mathrm{~W} / \mathrm{m}^{2}$ $\mathrm{K})$;

- $\quad$ windows with wooden frame and double-glazing (surface $=1.1 \mathrm{~m} \times 2.8 \mathrm{~m}$; thermal transmittance $=2.55 \mathrm{~W} / \mathrm{m}^{2} \mathrm{~K}$ );

It is worth mention that the selected configuration is the most representative both in terms of morphology and typology and in relation to the transformation degree - typically medium/low - of the envelope components.

The two models for the tower house are quite similar, except from the number of heat dispersant masonries, which are one surface in the middle unit and two surfaces in the end unit (Figure 6).
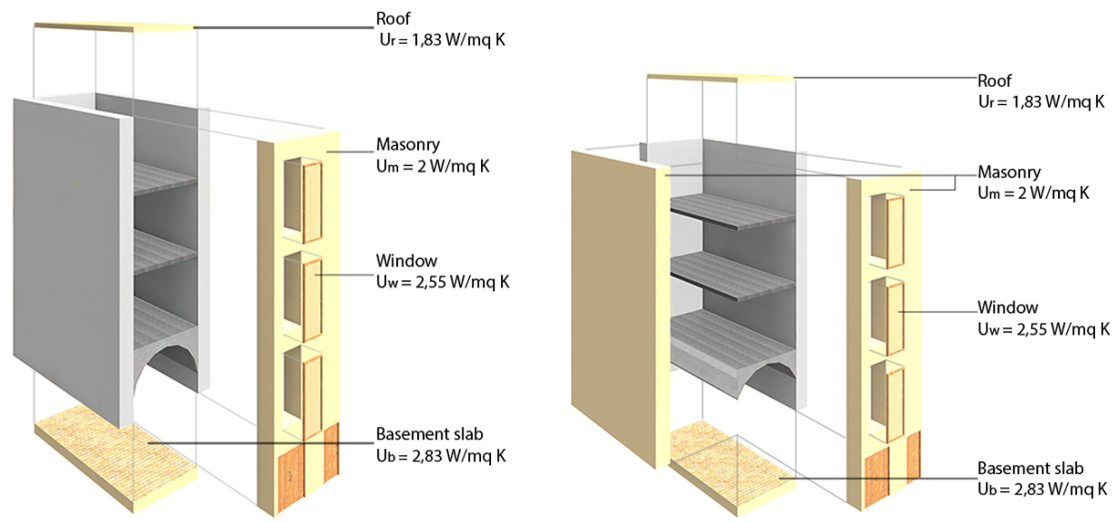

Figure 6: Heat dispersant sub-systems in "tower-house" models. 
The two models were assessed by heat transient analysis in order to estimate heating and cooling consumptions of the building-types. In detail, all the components were assumed as single-layer materials, with equivalent thermal transmittance of the actual multi-layers sub-systems. Then, the thermal transmittance of each component at a time was decreased, from the actual value to the normative threshold, through five intermediate steps, in order to assess the percentage incidence on the overall energy saving and, thus, identify the most influencing factors and the priorities of intervention.

As shown in the diagrams (Figure 7), the two models show quite comparable behavior. However:

- $\quad$ in the middle unit, the roof (R.M.T. wint) and the walls (W.M.T. wint) have similar impact on the heating loads, whereas the cooling loads are mainly affected by the roof performances. In fact, assuming that their thermal transmittance is decreased according to the normative thresholds $(\mathrm{U}=0.32$ $\mathrm{W} / \mathrm{mK}$ for the roof and $\mathrm{U}=0.34 \mathrm{~W} / \mathrm{mK}$ for the walls), the roof and the walls independently enable above $15 \%$ heating saving and respectively $14 \%$ and 4\% cooling saving;

- $\quad$ in the end unit, the main influencing components are the walls both in winter and summer (W.E.T. wint and W.E.T. sum). In fact, their compliance to the normative standards is responsible for $35 \%$ heating saving and $20 \%$ cooling saving, against about $10 \%$ in both seasons for the retrofitting of the roof.

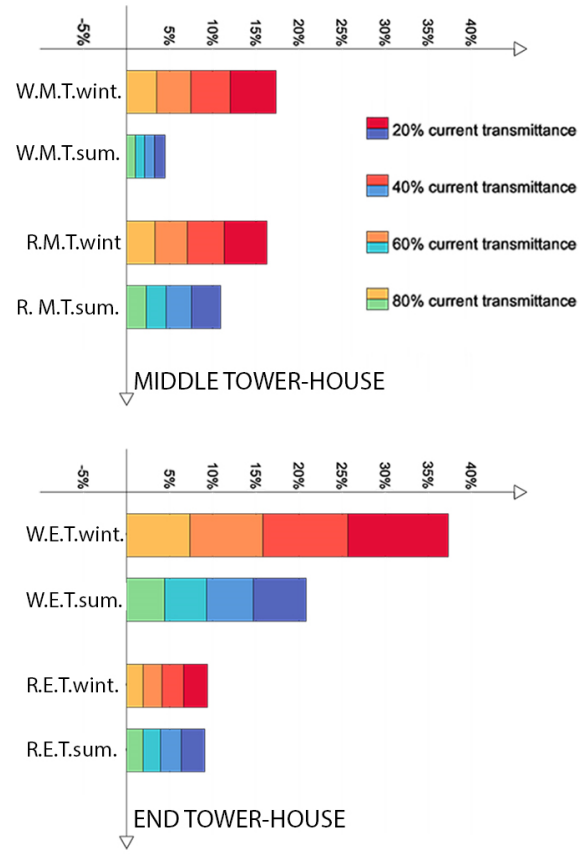

Figure 7: Thermal transmittance decrease vs. energy consumption saving in tower-house models. 
Thus, starting from those two components, their retrofitting was assessed against the above-mentioned transformation degree.

\subsection{Selection and validation of solutions}

In order to address the retrofitting solutions, in terms of both energy efficiency and low intrusiveness on the original building character, specific checklists were developed, comprising:

I. Sub-system under intervention;

II. Transformation degree;

III. Retrofitting construction systems and products, including traditional solutions and innovative materials under market development.

As far as the middle unit of the tower house is concerned, the following solutions were selected and validated.

a. A high-performing insulation material would retrofit the roof, in order to reduce the heating consumptions in wintertime, without significantly increase the component thickness and, thus, the terrace height. At the same time, a phase change material (PCM) would improve the performances in summertime. In fact, PCMs show high capability of thermal energy storage (or release) above (or below) a pre-set temperature, so that during the summer season, the benefits are mainly a decrease in overall energy consumption by the air conditioning unit and a time shift in peak load during the day. Specifically, a $40 \mathrm{~mm}$ aerogel panel was simulated to decrease the overall transmittance according to the normative threshold. Moreover, PCMs were simulated as outer layer above the waterproofing membrane, as thermal regulators of the outdoor temperature peak, assuming to incorporate phase change hydrates salts in water solutions for manufacturing $30 \mathrm{~mm}$ thick cement-based roof tiles. It is worth mention that alternative systems - e.g. less performing insulation panels or PCM boards under traditional roof tiles - were ruled out due to unacceptable morphological changes either/or poor performance improvements.

b. A high-performing insulation material on the internal side would retrofit the unplastered walls at the ground floor, in order to reduce the heating consumptions in wintertime, without significantly increase the component thickness and, thus, the indoor functional spaces. Among several materials (Table 1), aerogel and vacuum-insulation panels (VIPs) were mainly taken into account. However, aerogel was finally selected, because it is quite flexible and adaptable to irregular surfaces, whereas VIPs might undergo performance failures if the vacuum conditions are compromised during installation and utilization.

c. A high-performing insulation material on both sides would retrofit the plastered walls at the upper floors. Once more, aerogel boards resulted as the most performing solutions, in terms of both improvement of the energy performances and conservation of the historic and architectural features. 
Table 1: Thermal conductivity and thickness of the insulating panels.

\begin{tabular}{|c|c|c|}
\hline Type of insulation & Thermal conductivity (W/mK) & Thickness (mm) \\
\hline EPS & 0.036 & 90 \\
\hline Pressed wood & 0.04 & 100 \\
\hline VIP & 0.004 & 10 \\
\hline Aerogel & 0.015 & 40 \\
\hline
\end{tabular}

\section{Conclusions}

The validation of the described methodology for a representative historic town shows that the development of parametric models, describing the real case studies throughout assessment and intervention, might act as valuable decision-making support towards both energy efficiency and formal-material preservation.

Thus, the selection of compatible solutions for the energy retrofitting of the historic built heritage should rely on a geo-cluster vision, in order to represent the variety of environmental, architectural and constructional characters at the macroscale by a limited number of performance-based models. Those models should address assessment of actual energy behavior, identification of priorities of intervention and validation of conservative measures, taking into account the transformation degree of the building envelope and the technological development of innovative products and systems. That is required to support wide-ranging programs by public bodies and institutions for safeguard and conservation of the landscape and the cultural heritage, in terms of training, dissemination and incentivisation for all the involved stakeholders of the value chain, i.e. private citizens, enterprises and producers. Consequently, the qualification of traditional materials, techniques and technologies, as well as the validation of retrofitting solutions, should go beyond the case-by-case action towards the development of general guidelines, practice codes and databases, with methodological robustness and operational flexibility.

\section{References}

[1] Project FP7-NMP. GE2O "Geo-clustering to deploy the potential of Energy Efficient Buildings across EU”. www.geocluster.eu

[2] Lombardo G., Cicero C., Rehabilitation strategies for energy-efficient buildings in GeoCluster. Advances in Building Energy Research. Taylor \& Francis, 2014.

[3] Pizzi E., De Tommasi G., Guida A.G.M.I.R., Morganti R.T.G., Salemi A., (2013), Large Scale Building Refurbishment Strategies in Italy: A Proposal of "Geocluster" Characterization, Proc. of 39th World Congress on Housing Science, Milan, September 17-20, 2013.

[4] Cem Doğan Şahin, Zeynep Durmuş Arsan, Selim Sarp Tunçoku, Tor Broström, Gülden Gökçen Akkurt, A transdisciplinary approach on the 
energy efficient retrofitting of a historic building in the Aegean Region of Turkey, Energy and Buildings, Available online 14 March 2015.

[5] Shaoqing Gou, Zhengrong Li, Qun Zhao, Vahid M. Nik, Jean-Louis Scartezzini, Climate responsive strategies of traditional dwellings located in an ancient village in hot summer and cold winter region of China, Building and Environment, Volume 86, pp. 151-165, 2015.

[6] Endrik Arumägi, Targo Kalamees, Analysis of energy economic renovation for historic wooden apartment buildings in cold climates, Applied Energy, Volume 115, pp. 540-548, 2014.

[7] European Commission "Energy-efficient buildings PPP. Multi-annual roadmap and longer term strategy", Luxembourg: Publications Office of the European Union, 2010.

[8] European Commission "Energy-efficient buildings. Multi annual roadmap for the contractual PPP under Horizon 2020", Luxembourg: Publications Office of the European Union, 2013.

[9] William Chung, Review of building energy-use performance benchmarking methodologies, Applied Energy, Volume 88, Issue 5, pp. 1470-1479, 2011.

[10] W.L. Lee, Benchmarking energy use of building environmental assessment schemes, Energy and Buildings, Volume 45, pp. 326-334, 2012.

[11] Ayşegül Tereci, Soofia Tahira Elias Ozkan, Ursula Eicker, Energy benchmarking for residential buildings, Energy and Buildings, Volume 60, pp. 92-99, 2013.

[12] Zhengwei Li, Yanmin Han, Peng Xu, Methods for benchmarking building energy consumption against its past or intended performance: An overview, Applied Energy, Volume 124, 1, pp. 325-334, 2014.

[13] Project IEE TABULA “Typology Approach for BUiLding stock energy Assessment". http://episcope.eu/iee-project/tabula/

[14] Project IEE EPISCOPE “Energy Performance Indicator Tracking Schemes for the Continuous Optimisation of Refurbishment Processes in European Housing Stocks". http://episcope.eu/iee-project/episcope/

[15] De Berardinis P., Rotilio M., Marchionni M., Friedman A., Improving the energy-efficiency of historic masonry buildings. A case study: A minor centre in the Abruzzo region, Italy, Energy and Buildings, Volume 80, pp. 415-423, 2014. 